\title{
Das Entwerfen von Bandkarten des internationalen Handels
}

Bandkarten, im Englischen "flow charts" genannt, werden in wirtschaftsgeographischen Arbeiten bevorzugt zur Darstellung weltwirtschaftlicher Beziehungen verwendet, weil sie sich durch $\mathrm{Klar}$ heit und einen hohen Informationsgehalt auszeichnen. Im folgenden sollen einige Probleme besprochen werden, mit denen sich ein Kartenautor beim Entwurf solcher Karten auseinanderzusetzen hat; dabei handelt es sich sowohl um die statistischen Grundlagen wie die Technik der zeichnerischen Gestaltung. Ausserdem werden wir im Laufe der Besprechung sehen, dass sich das Zeichnen solcher Bandkarten aus didaktischen Gründen ausgezeichnet als Thema in Uebungen zur Kartenkunde eignet. Selbst dann, wenn die statistischen Rahmenbedingungen für alle Teilnehmer die gleichen sind, kann ein Studierender den Entwurf immer noch in hohem Masse individuell und kreativ gestalten. Die hier beigegebene Bandkarte "Bauxit und Aluminium" wurde in einem solchen Kurs des Geographischen Institutes der Universität Zürich (Leitung PD Dr. H. Kishimoto) von $H$. Litz gezeichnet.

Bandkarten gehören, in einen grösseren Zusammenhang hineingestellt, zur Kategorie der graphischen Darstellungen von Systemen. Nach Definition besteht ein System aus einer endlichen Zahl von Elementen (in diesem Falle Länder oder Ländergruppen) und den zwischen diesen bestehenden Beziehungen (Güterströme, Handelsverkehr). Wir könnten ein System natürlich auch verbal beschreiben, mathematisch festlegen oder in einer Matrixform ausdrücken; die kartographische Darstellung drängt sich im Falle des internationalen Handels von der Sache her gesehen auf, handelt es sich doch um räumliche Beziehungen und Verteilungsmuster. Ausserdem ist die Sprache der Graphik international und auch für Laien ohne Schwierigkeiten verständlich.

Bandkarten (wir sprechen hier und im folgenden immer nur von der Darstellung weltwirtschaftlicher Beziehungen) können wir vorerst nach ihrem Generalisierungs- oder Abstraktionsgrad unterteilen. Die Elemente des Systems sind im Falle der grössten möglichen Detaillierung die einzelnen Länder der Erde, so wie sie die Statistik der Vereinten Nationen aufführt. Im Zuge fortschreitender Generalisierung können diese zu Regionen und schliesslich zu den drei von den Vereinten Nationen unterschiedenen Hauptkategorien: I. Marktwirtschaftliche Länder: (a) entwickelte, (b) in Entwicklung begriffene, II. Länder mit zentral geplanter Wirtschaft, zusam- mengefasst werden. Bei starker Generalisierung kann das System schematisch in Form eines Kartogramms abgebildet werden; gliedert man jedoch bis zum einzelnen Lande auf, verwendet man als Bezugsnetz zweckmässig eine geeignete Projektion einer Weltkarte.

Im Zusammenhang mit wirtschaftsgeographischen Aufgaben brauchen wir in kartographischen Uebungen üblicherweise ein flächentreues Netz. Wir besassen schon eine grosse Zahl von Andrucken der flächentreuen Briesemeisterprojektion und verwendeten sie aus diesem Grunde für die Karte "Bauxit und Aluminium". Dieses Netz hat freilich den Nachteil, dass es im Pazifik aufgeschlitzt ist; im Beispiel "Bauxit und Aluminium" ist dies nicht gravierend, während bei anderen Sachverhalten eine Projektion mit dieser Eigenschaft denkbar ungeeignet wäre. Bevor man mit der eigentlichen Arbeit beginnt, muss in jedem Falle die Wahl eines zweckmässigen Bezugsnetzes sorgfältig überlegt werden. Ohne Zweifel würden wir dann nach einem besseren Netz als Briesemeister suchen.

Bandkarten können wir auch in analytische (nur einzelne Güterkategorien) und synthetische oder zusammenfassende (gesamter Aussenhandel) gliedern. Sowohl die statistischen Probleme wie die Technik der Kartenbearbeitung sind für die beiden Gruppen in mancher Hinsicht verschieden.

Die statistischen Rohdaten werden in jedem Falle durch die Zollbehörden dann ermittelt, wenn die aus- oder eingeführten Waren der Deklaration unterliegen. Dabei wird festgestellt: Warenkategorie, Wert, in vielen Fällen auch Menge/Gewicht sowie entsprechend den Vorschriften der einzelnen Länder das Bestimmungs- oder Herkunftsland. Wenn wir uns vorerst den synthetischen Karten zuwenden, spielen alle Fragen, welche die Warenkategorie betreffen, keine Rolle. Der Umstand, dass in zahlreichen Fällen nur der Wert, nicht aber die Menge deklariert wird, zwingt uns, für Bandkarten den Wertmassstab zu verwenden. Dies ist zu bedauern, weil in der Wirtschaftsgeographie im Hinblick auf verkehrsgeographische Fragestellungen oft Gewicht oder Volumen sinnvoller wären; die Statistik der Vereinten Nationen folgt jedoch - genau so wie die Zollstatistik der einzelnen Länder - in erster Linie volkswirtschaftlichen. Ueberlegungen. Ein besonderes

Prof. Dr. H. Boesch, Geographisches Institut der Universität Zürich, Blümlisalpstr. 10, 8006 Zürich. 
Problem stellt die Festlegung von Herkunftsund Bestimmungsland dar. Weil oft zwei Länder $A$ und $B$ trotz aller Bemuihungen der Vereinten Nationen um eine Koordinierung diese Begriffe auch heute noch sehr verschieden definieren, stimmen die Angaben über Ausfuhren von $A$ nach $B$ im allgemeinen mit den Einfuhren von $B$ aus A nicht überein. Diese Differenzen, die sehr beträchtlich sein können, verschwinden, wenn wir Ausfuhren und Einfuhren für alle Länder zum Welttotal aufaddieren. Uebrig bleibt freilich auch dann noch eine Differenz: Die Welttotale der Ausfuhren sind immer etwa 5 bis 7 Prozent niedriger als jene der Einfuhren. Der Grund für diese Differenz liegt darin, dass der Einfuhrwert auch noch die Kosten der Transportversicherung und der Transportkosten einschliesst. Man spricht bei den Ausfuhrwerten von fob (free on board)-Preisen, bei den Einfuhren von cif (cost + insurance + freight)-Preisen. Neben den zwei erwähnten Gründen gibt es noch andere Ursachen auftretender Differenzen; ihre Besprechung im einzelnen würde jedoch hier $z u$ weit fuihren. Ein weiteres Problem bildet schliesslich die Umrechnung der in Landeswährung festgestellten Aussenhandelswerte in eine geme insame Währung (beispielsweise US-Dollar); dem Kartenautor wurde diese Arbeit glücklicherweise vom Statistischen Bureau der Vereinten Nationen abgenommen. Die Zeichnung von Bandkarten wäre kaum möglich, wenn das von den einzelnen Ländern den Vereinten Nationen gelieferte Zahlenmaterial von ihnen nicht aufgearbeitet und in geeigneter Form veröffentlicht worden wäre. Unter den Publikationen der Vereinten Nationen sind für den vorliegenden Zweck das jährlich erscheinende Statistical Yearbook und das ebenfalls jährlich erscheinende Yearbook of International Trade Statistics die wichtigsten.

Handelt es sich darum, den gesamten Aussenhandel darzustellen, enthalten diese Veröffentlichungen das statistische Ausgangsmaterial entsprechend dem gewünschten Generalisierungsgrad in verschiedener Anordnung. Bei grösster Detaillierung (d.h. nach einzelnen Ländern aufgegliedert) finden wir im Yearbook of International Trade Statistics für jedes Land eine Tabelle, welche für eine genügend grosse Zahl von Handelspartnern die Aus- und Einfuhrtotale enthält. Fassen wir jedoch Länder zu Regionen und schliesslich in den drei erwähnten Hauptkategorien zusammen, finden wir die statistische Information ausserdem in Matrixform, was ein müheloses und rasches Konstruieren von Kartogrammen gestattet.

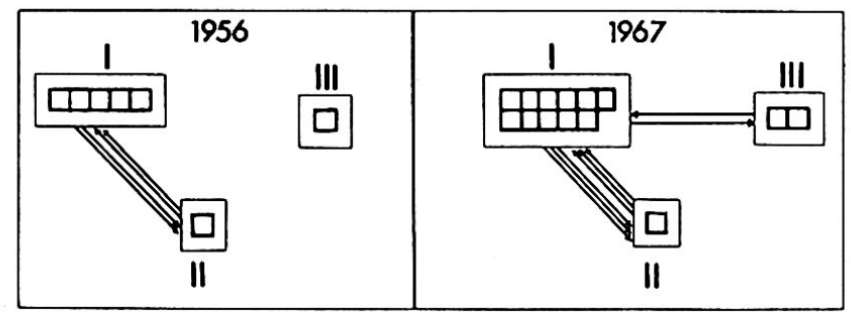

Mühsam und vor allem zeitraubend ist nach dem Gesagten das Zeichnen einer Bandkarte des internationalen Handels, wenn wir alle Länder berücksichtigen, weil in diesem Falle eine Matrixtabelle, die einen raschen Ueberblick über die in die Zehntausende gehenden Beziehungen geben würde, fehlt. Wir müssen aus den erwähnten Länderstatistiken (jeweilen Tab. I im Yearbook of International Trade Statistics) jede einzelne Beziehung herauslesen. Dabei verwenden wir grundsätzlich nur die Exportwerte f.o.b. Als erster wichtiger Schritt ist festzulegen, welches Grundquantum $Q$ noch berücksichtigt werden soll; Werte unter $0,5 \mathrm{Q}$ bleiben in der Folge unberücksichtigt. Wir verfügen damit über ein Mittel, bei konstanter Elementenzahl des Systems die Verbindungen zu generalisieren und gleichzeitig den Arbeitsaufwand zu reduzieren. Ein zeichnerisches Vorgehen bei der Uebertragung der festgestellten Beziehungen auf die Grundkarte erweist sich wegen des bald unentwirrbaren Netzes von Linien rasch als unmöglich. Dagegen hat sich die folgende Technik bewährt: Man beginnt von einem Lande $A$ aus nach allen jenen Ländern, mit denen ein Export von $Q$ oder einem Vielfachen davon besteht, eine entsprechende Anzahl von Fäden zu spannen. Anschliessend wird für alle Länder B,C ....n gleich verfahren; so wird in einem ersten Arbeitsgange die Gesamtheit aller zu berücksichtigenden Beziehungen durch die gelegten Fäden festgehalten. Dieser erste Schritt ist eine langwierige und im ganzen rein mechanische Arbeit. In einem zweiten Schritt müssen die Fäden geordnet werden. Die Festlegung der Ausfuhrhäfen und der Transportrouten stellt uns dabei vor schwierige Probleme. Natürlich kann man auch hier mehr oder weniger generalisieren, doch sollte in einer guten Bandkarte beispielsweise ersichtlich sein, welchen Anteil die verschiedenen Küstenabschnitte der USA oder der UdSSSR am Landestotal haben. Neben Hafenstatistiken hilft hier - und damit wird das Entwerfen einer Bandkarte geographisch anspruchsvoll nur noch das Studium von Spezialliteratur, das richtige Kombinieren und die Erfahrung weiter. Bei der Festlegung der Transportrouten helfen gewisse Kontrollpunkte (z.B. Panamakanal-Statistik); deren Zahl ist aber klein. Im dritten Schritt werden, nachdem alle Fäden geordnet und weitgehend gebündelt worden sind, die in jedem Abschnitt vorhandenen Fäden gezählt und auf dieser Grundlage die Bänder gezeichnet. Damit werden die Zusammenhänge immer klarer und wir können uns nun den graphischen und kartographischen Fragen zuwenden.

Fig. 1: Stark generalisierte Darstellung der weltwirtschaftlichen Bezighungen. Der Signaturwert beträgt 10 Milliarden US S. Es bedeutet: (I) Marktwirtschaft, Industrieländer, (II) Marktwirtschaft, Entwicklungsländer, (III) Planwirtschaft. Pfeile stellen den Handel zwischen I, II und III dar, während Quadrate den internationalen Handel innerhalb einer der drei Gruppen repräsentieren. (aus Boesch H., weltwirtschaftsgeographie, 1977) 


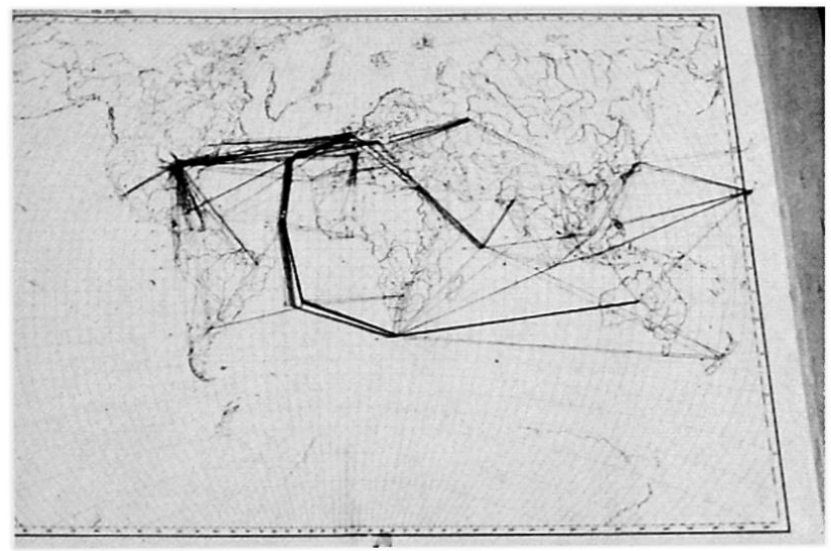

Fig. 2: Welthandel 1958, abgesteckte Fadenkarte des Entwurfes; Ausführung $I$. Kutschke.

Die Zahl der Verbindungen ist bei analytischen Bandkarten (oder anders ausgedrückt: bei Teilsystemen) sehr viel kleiner als bei synthetischen Bandkarten (anders ausgedrückt: im Falle des gesamten Aussenhandels). Aus diesem Grunde kann man bei den letztgenannten Karten in den meisten fällen auf das reichlich muihsame Verfahren des Fadenspannens verzichten und direkt von der Handelsmatrix aus zum Zeichnungsentwurf schreiten.

Bis zu diesem Punkte befassten wir uns nur mit den Beziehungen zwischen den Knotenpunkten. Um das "System Welthandel" kartographisch zu erfassen, müssen zusätzlich noch die relevanten Eigenschaften der Länder berücksichtigt werden. Die statistisch mögliche Menge von Darstellungen wäre gross, die Kartengraphik zwingt uns jedoch zu einer strengen Auswahl. Eine überladene Karte wird unleserlich und vermag die grossen $\mathrm{Zu}$ sammenhänge nicht mehr klar zu zeigen. Im allgemeinen sollten wir uns auf zwei zusätzliche Aussagen beschränken, wobei eine als Signatur (Symbol) und die andere als Flächenraster wiedergegeben werden kann.

Die graphische Gestaltung hängt im übrigen sehr davon $a b$, ob die fertige Bandkarte für eine politische Broschüre, ein volkswirtschaftliches Lehrbuch, ein Schulbuch der Oberstufe usw. bestimmt ist. In diesem letzten und mit Bezug auf die individuelle Gestaltungsfreiheit wichtigsten Schritt gibt man bei Uebungsaufgaben den Studierenden die Möglichkeit, ihre eigenen Auffassungen zu realisieren - gleichzeitig aber auch erhält der Uebungsleiter manche Auskunft über ihre Persönlichkeit. Ueblicherweise schliesst sich an diese Arbeitsphase auch eine interessante Diskussion zur Frage, ob im Schulunterricht sehr detaillierte oder eher abstrakte Karten bevorzugt werden, an. Neuere Schulatlanten lassen vermuten, dass ein möglichst reicher Informationsgehalt einer das Wesentliche herausarbeitenden Generalisierung vorgezogen wird. Die Ansichten der Lehrer sind aber nach unserer Erfahrung in dieser Frage doch sehr geteilt.
Bei analytischen Karten (unter diesem Begriff wollen wir Bandkarten verstehen, die bestimmte Warenkategorien betreffen) ergeben sich zusätzliche Probleme.

Die wichtigste Voraussetzung solcher Karten ist eine einheitliche Definition der Warenkategorien. Mit der revidierten Standard International Trade Classification (SITC, revised, UN publication, sales no. 51XVII.1) wurde dieses Ziel teilweise erreicht. Die SITC gliedert alle in Frage kommenden Positionen im Aussenhandel in Sektionen (10), Abteilungen (56), Gruppen (177), Untergruppen (625) und einzelne Güter oder "items" (1312). Jede Kategorie wird durch einen Zahlencode bezeichnet, der ein, zwei oder mehr "digits" enthält. Beispielsweise gehört zur 3-digits Kategorie die Gruppe (011)=Fleisch, frisch, gekühlt und gefroren. Für die beiden Ländergrupoen im marktwirtschaftlichen Sektor (Industrie-und Entwicklungsländer), die beide die SITC befolgen, ist eine Zusammenfassung der von den einzelnen Ländern gelieferten Daten möglich. Für die Länder mit zentral geplanter Wirtschaft, die den Vorschriften der SITC nicht nachleben, fehlen dafür die Voraussetzungen.

Die Standardisierung der Warenkategorien ist aber nur ein erster und für unsere Zwecke noch ungenügender Schritt. In den Länderstatistiken des Yearbook of International Trade Statistics finden sich nämlich nur statistische Angaben über die Güterein- und Güterausfuhr jedes einzelnen Landes, nicht aber über den Handel zwischen den einzelnen Ländern. Früher gaben in wenig befriedigender Form (vor allem weil nur eine beschränkte Zahl von Ländern berücksichtigt wurde) die Commodity Trade Statistics des Statistischen Büros der Vereinten Nationen über den zwischenstaatlichen Handel Auskunft. Seit wenigen Jahren erscheinen nun erfreulicherweise im Yearbook of International Trade Statistics für sehr zahlreiche 3-digit Kategorien übersichtliche und vollständige Matrixtabellen. Sie haben nur den einen Nachteil, dass auch hier die kommunistischen Länder (Kategorie der Länder mit zentral geplanter Wirtschaft) unberücksichtigt bleiben mussten. Nur der Handelsverkehr der marktwirtschaftlichen Länder mit dieser Gruppe konnte in einer Marginalangabe (ausserhalb der Matrix) über den Gesamtwert des Handelsverkehrs mitberücksichtigt werden. In die Matrix selbst sind aber lediglich die marktwirtschaftlichen Länder aufgenommen worden. Es gibt Fälle, wo das Fehlen von Angaben über die kommunistischen Länder nicht ins Gewicht fällt, während in andern Fällen die Möglichkeit besteht, über die nationalen Statistiken die Lücken einigermassen zu schliessen. Vielleicht wird sich ein Kartenautor auch dafür entscheiden müssen, alle planwirtschaftlichen Länder weiss zu lassen und dies in der Legende entsprechend zu begründen.

Ausserhalb der eigentlichen Matrix finden sich im Titel jeder Tabelle die folgenden Angaben: SITC-Code, Güterkategorie, Jahr, Totale der Ein- und Ausfuhren aller marktwirtschaftlichen Länder unter sich und mit den Handelspartnern 


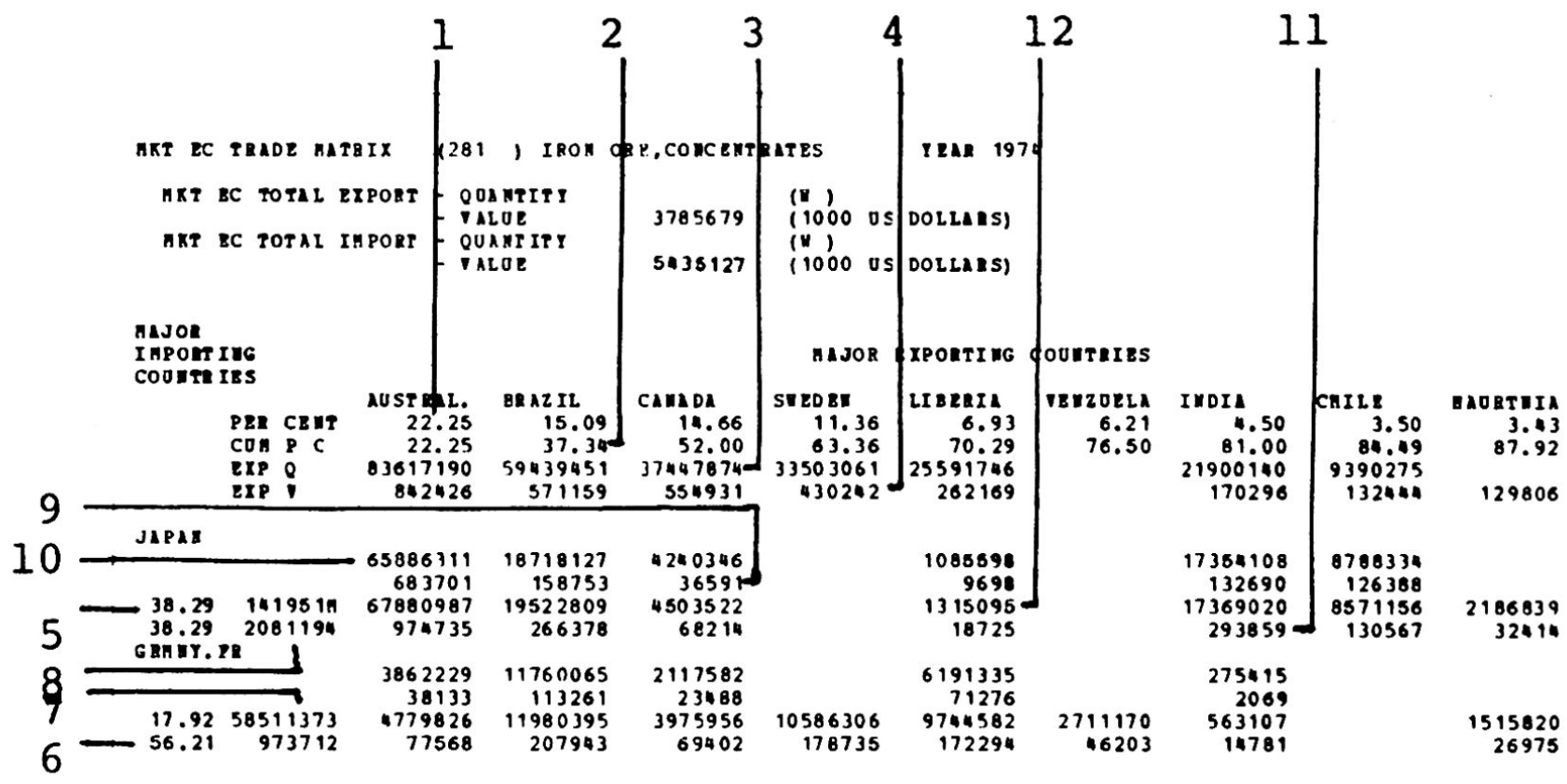

Fig. 3 : Ausschnitt aus der Handelsmatrix für S.I.T.C. 281, Eisenerz u. Konzentrate (siehe Text)

unter den planwirtschaftlichen Ländern sowohl nach Wert und Menge (diese Position bleibt aus den genannten Gründen offen).

Die Matrix selbst verzeichnet in der Kopfzeile von links nach rechts angeordnet die 22 wichtigsten Exportländer in absteigender Reihenfolge sowie in den Säulen von oben nach unten in gleicher Weise die wichtigsten Importländer. In der Regel genügt dies, um rund 90 , oft aber auch mehr als 95\% der Handelsbeziehungen zu erfassen. Jedem Ausfuhrland wird zugeschrieben (die Zahlen beziehen sich auf die Einzeichnungen auf dem hier wiedergegebenen Ausschnitt einer Handelsmatrix): (1) Prozentanteil am oben erwähnten Exporttotal, (2) in der Zieile aufaddierter Prozentanteil, (3) Exportmenge nach Angabe des Exportlandes, (4) Exportwert nach Angabe des Exportlandes. In gleicher Weise wird jedem Einfuhrland zugeschrieben: (5) Prozentanteil am oben erwähnten Importtotal, (6) in der Säule aufaddierter Prozentanteil, (7) Importmenge nach Angabe des Importlandes, (8) Importwert nach Angabe des Importlandes. Im entsprechenden Feld der Matrix finden sich die folgenden Angaben: (9) Wert der Ausfuhren in das betreffende Einfuhrland nach Angaben des Ausfuhrlandes, (10) dasselbe, aber Menge, (11) Wert der Einfuhren aus dem betreffenden Ausfuhrland, nach Angaben des Einfuhrlandes, (12) dasselbe, aber Menge. Ein Vergleich dieser Werte zeigt, wie gross die Differenzen zwischen den Angaben von Aus- und Einfuhrland sein können. Da viele Länder die Güter nur wertmässig erfassen, fehlen oft die Angaben für 3,7,10 und 12 .
Für die Karte "Bauxit und Aluminium" konnte aber auch diese neue Handelsmatrix nicht weiterhelfen, weil Bauxit einer 4-digit Kategorie (SITC 2833; 283 bedeutet Erze und Konzentrate von Grundnichteisenmetallen) angehört und damit nicht in die Matrix aufgenommen wurde. In diesem Falle hilft jedoch eine andere Quelle weiter. Jährlich wird von der OECD unter dem Titel les Transports Maritimes eine Broschüre veröffentlicht, welche neben zahlreichen anderen Informationen für wichtige Güter (Kohle, Erdöl, Eisenerz, Getreide, Phosphat, Bauxit usw.) eine entsprechende Handelsmatrix enthält. Die Angaben erfolgen hier verständlicherweise in Gewichtsmengen; die Elemente des in der Matrix wiedergegebenen Systems sind nur in seltenen Fällen einzelne Länder, meistens aber Regionen. Zusammen mit den Länderstatistiken der erwähnten Jahrbiicher der Vereinten Nationen bildete diese OECD-Veröffentlichung die statistische Grundlage beim Entwurf der Karte. Auch analytische Karten sollten zusätzlich zu den Bändern Informationen über die Elemente (oder Knoten des Systems) enthalten und wiederum muss mit Sorgfalt geprift werden, was mit den beschränkten kartographischen Möglichkeiten sinnvoll dargestellt werden soll. Die Karte "Bauxit und Aluminium" beschränkt sich auf die quantifizierte Wiedergabe der entsprechenden Produktion, die mit $\mathrm{Hilfe}$ von Zusatzliteratur lagerichtig eingezeichnet wurde. Möglich wäre irgend eine weitere Angabe als Flächenraster gewesen; offenbar drängte sich dem Bearbeiter keine relevante Information auf und er beschränkte sich auf das Genannte. 


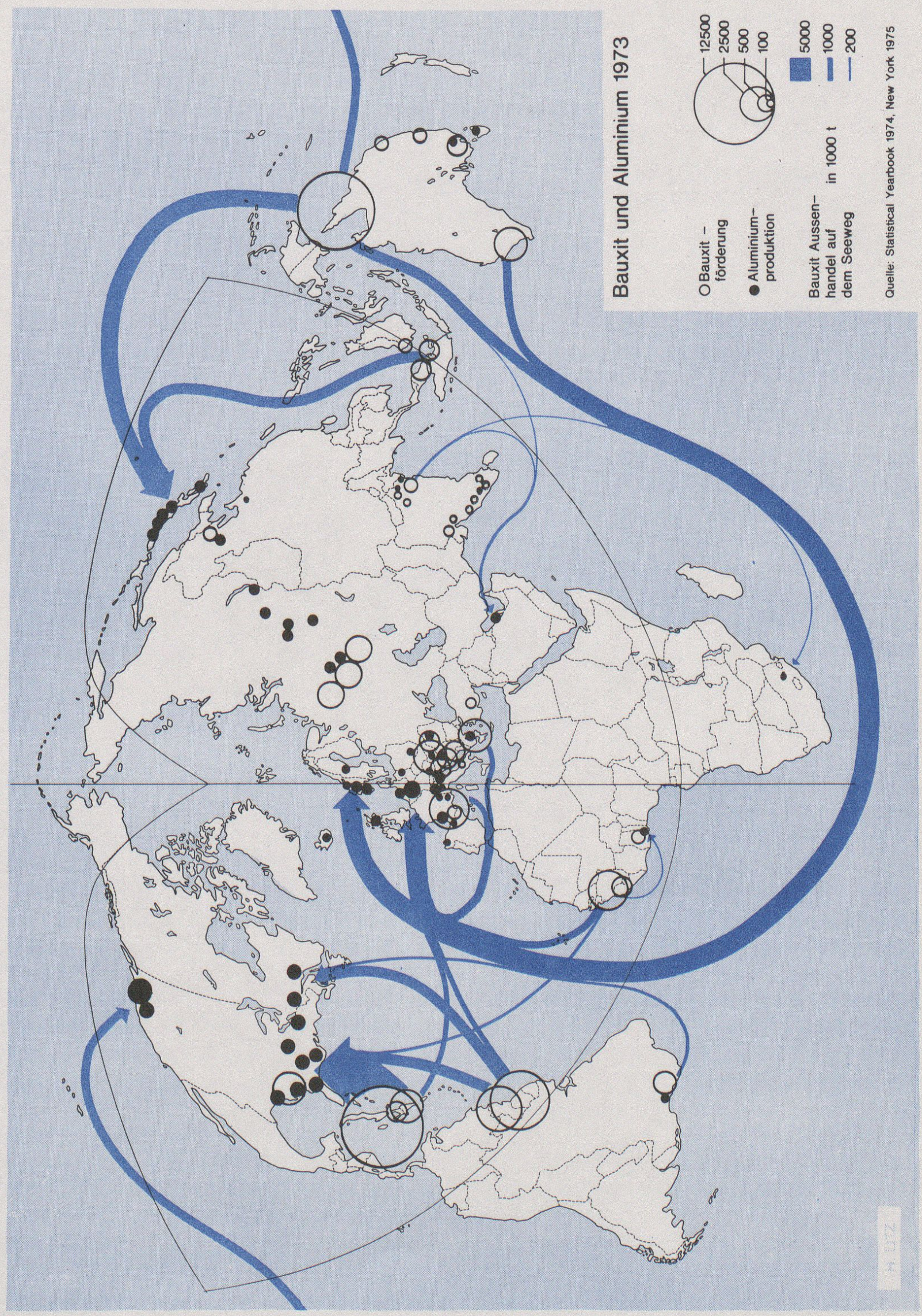

Fig.4 Beispiel einer Bandkarte : Bauxit und Aluminium ; Entwurf H.Litz . 